\title{
Exercise-induced vasculitis
}

\author{
Philipp Jud MD, Franz Hafner MD
}

Cite as: CMAJ 2018 February 20;190:E195. doi: 10.1503/cmaj.171377

A previously healthy 61 -year-old woman presented to the outpatient clinic for angiology with a rash on both lower legs after a strenuous hiking tour. The patient had not been injured and there was no itch or pain associated with the rash. She had no arthralgias, abdominal pain, fever, malaise or weight loss. The patient was not taking any medication. A similar episode, which occurred after a hiking tour a few years previously, resolved spontaneously within one week. On examination, she had slight edema and petechiae on both lower legs without ulceration; an area of skin compressed by the sock cuff was spared bilaterally (Figure 1). Digital pulse oscillography and doppler ultrasonography of the vessels of the lower limb were normal. Results for her complete blood cell count, renal function, C-reactive protein, erythrocyte sedimentation rate, serum protein electrophoresis, antineutrophil cytoplasmic antibodies, subtypes of antinuclear antibodies, C1q antibodies, cryoglobulins and complement factors were within physiologic ranges. We did not find any coagulopathy. We concluded that this was exercise-induced vasculitis. The patient's symptoms resolved within six days without treatment.

Exercise-induced vasculitis is a small-vessel vasculitis affecting the lower legs that occurs after strenuous sports activity such as running or hiking, particularly during hot weather. It is more common in women over 50 years of age and presents with petechial or urticarial rash with edema, itch or pain in an otherwise healthy patient. The skin area compressed by the sock cuff is characteristically spared. ${ }^{1}$

Sports activity in hot weather may increase circulating immune complexes and complement activation, ${ }^{2}$ which leads, together with muscle fatigue and impairment of venous drainage, to the development of petechiae and vasculitis. A skin biopsy may show leukocytoclastic vasculitis. ${ }^{1,3}$

Exercise-induced vasculitis is self-limiting and resolves after a few days. Nonsteroidal antiinflammatory drugs, topical corticosteroids and elevation of the affected legs may reduce symptoms. ${ }^{3}$ Although a patient's history and clinical findings may suggest exercise-induced vasculitis, any investigation should exclude autoimmune disorders or coagulopathies.

\section{References}

1. Kelly RI, Opie J, Nixon R. Golfer's vasculitis. Australas J Dermatol 2005;46:11-4.

2. Nieman DC, Berk LS, Simpson-Westerberg M, et al. Effects of long endurance running on immune system parameters and lymphocyte function in experienced marathoners. Int J Sports Med 1989;10:317-23.

3. Espitia O, Dréno B, Cassagnau E, et al. Exercise-induced vasculitis: a review with illustrated cases. Am J Clin Dermatol 2016;17:635-42.

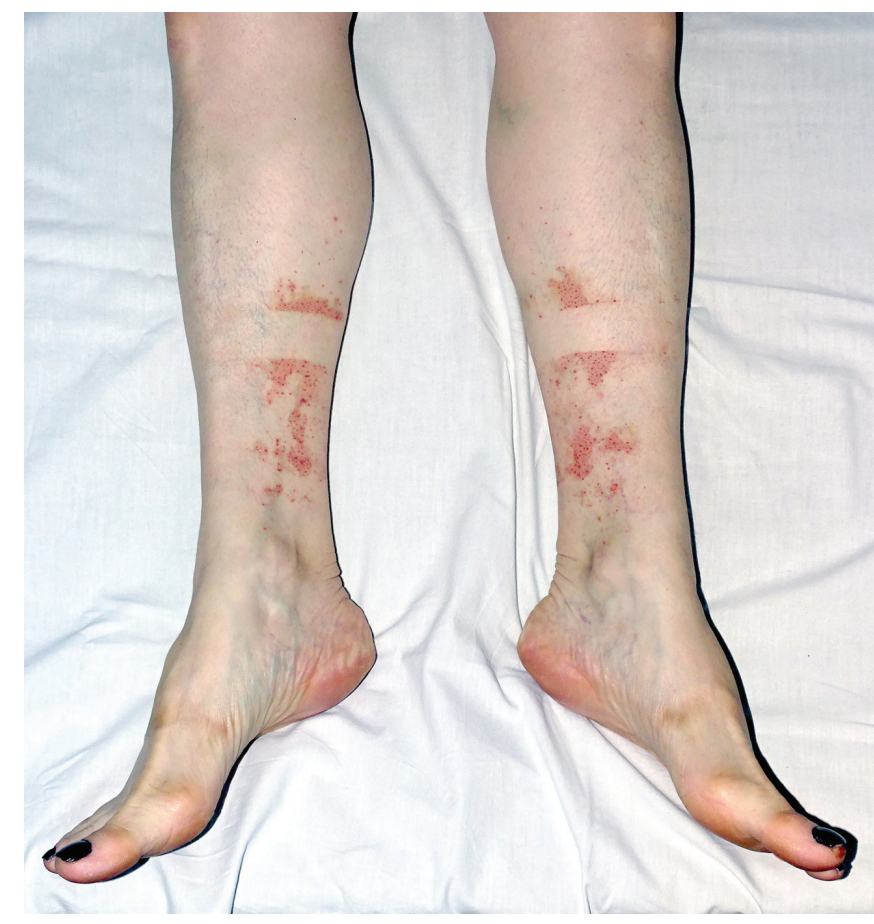

Figure 1: Petechial skin rash on the medial lower legs of a 61-year-old woman who had just finished a strenuous hiking tour. The area of skin that was compressed by the sock cuff was spared.

\section{Competing interests: None declared}

This article has been peer reviewed.

The authors have obtained patient consent.

Affiliation: Division of Angiology, Department of Internal Medicine, Medical University of Graz, Graz, Austria

Correspondence to: Philipp Jud, philipp.jud@medunigraz.at

Clinical images are chosen because they are particularly intriguing, classic or dramatic. Submissions of clear, appropriately labelled highresolution images must be accompanied by a figure caption. A brief explanation ( 300 words maximum) of the educational importance of the images with minimal references is required. The patient's written consent for publication must be obtained before submission. 